\title{
Impact of Appointment-Based Medication Synchronization on Existing Users of Chronic Medications
}

\author{
David Holdford, RPh, MS, PhD, FAPhA, and Kunal Saxena, BS, MS
}

\section{ABSTRACT}

BACKGROUND: Appointment-based medication synchronization (ABMS) has been associated with greater patient adherence and persistence when patients begin taking chronic medications. It is not known whether similar results will be seen for patients who have been taking chronic medications for 6 months or more.

OBJECTIVE: To compare the impact of a community pharmacy chain's ABMS program on medication adherence and persistence of existing users of chronic medications with individuals who are not enrolled in the program.

METHODS: A retrospective cohort study compared patients receiving ABMS with matched comparison groups receiving usual care. ABMS consisted of synchronizing a patient's medications to be dispensed on a single appointment day every month, a call to the patient prior to the appointment day to address any prescription changes and to remind the patient, and a patient visit to the pharmacy to pick up the medications. Outcomes were 1-year adherence rates using proportion of days covered (PDC) and 1-year nonpersistence rates. Data for this study came from prescription claims records of patients taking 1 of 6 chronic medication classes during the period of December 1, 2011, to February 28, 2014. ABMS patients were matched with comparison groups according to prior adherence behavior, medication class, age, gender, and geographic region.

RESULTS: Mean PDC scores ranged from 0.73 to 0.91 for ABMS patients ( $n=205$ to 716 ) and from 0.57 to 0.71 for usual care depending on the medication class. The percentage of adherent individuals (i.e., $P D C \geq 0.80$ ) was $55 \%$ to $84 \%$ for ABMS participants and $37 \%$ to $62 \%$ for usual care. Odds of adherence was 2.3 to 3.6 times greater with ABMS. Usual care patients became nonpersistent $(61 \%$ to $74 \%)$ more often than ABMS patients (33\% to $44 \%$ ) with hazard ratios of nonpersistence being 0.39 to 0.67 for individuals in the program.

CONCLUSIONS: An ABMS program in a community pharmacy setting was associated with higher rates of adherence and persistence for patients who had been taking chronic medications for at least 6 months. Approximately 18 to 35 additional ABMS participants were adherent for every 100 patients enrolled when compared with usual care. For every 100 patients receiving usual care, 17 to 40 additional patients in the ABMS group were persistent. This study shows that ABMS programs can improve medication adherence and persistence for patients who are newly prescribed or currently taking chronic medications.

J Manag Care Spec Pharm. 2015;21(8):662-69

Copyright $\odot 2015$, Academy of Managed Care Pharmacy. All rights reserved.

\section{What is already known about this subject}

Adherence to prescribed chronic medications is a major public health concern.

Medication nonadherence and nonpersistence lead to unsuccessful therapy outcomes, negative health effects, and huge economic burden.

Patient-centric interventions such as appointment-based medication synchronization (ABMS) have shown significant success in improving patient adherence and persistence to chronic medications in newly enrolled patients (new medication users).

\section{What this study adds}

ABMS was associated with greater adherence and persistence in patients who had been taking chronic medications for at least 6 months (existing medication users).

After controlling for prior adherence behavior between ABMS and non-ABMS patients, or the "healthy adherer" effect, the results still showed significant improvement in adherence and persistence in ABMS patients compared with non-ABMS patients.

A dherence and persistence with prescribed chronic medications is a major public health concern in the United States and worldwide. Acute and chronic medication therapies are only valuable in achieving positive health outcomes if patients adhere to and persist with their therapeutic plans. ${ }^{1}$ Failure to do so leads to negative health consequences and annual costs in the billions of dollars. ${ }^{2-6}$

Extensive research over time has shown that adherence and persistence with prescribed plans is an intractable problem. Despite numerous interventions by health care providers in a variety of settings, patients follow prescribed plans only $25 \%$ of the time. ${ }^{7}$ When prescribed chronic medications, patients adhere roughly $50 \%$ of the time. ${ }^{8-11}$ Improving adherence to prescribed medication may have greater impact on population health than any other specific medical treatment. ${ }^{12}$

Nonadherence to medications is a complex and multifactorial problem that is not easily resolved by simple solutions. ${ }^{1,12,13}$ The causes of nonadherence vary depending on individual characteristics (e.g., physical impairments, cognitive problems, age-related concerns, and belief about medicines); the patient's medical condition (e.g., symptoms and comorbidities); health system limitations (e.g., disjointed care and inaccessibility); 
therapeutic regimen complexity and associated adverse effects; and socioeconomic concerns (e.g., affordability, low reading and health literacy, and weak social support). ${ }^{1}$ This situation requires interventions that are tailored to patient needs and circumstances using various strategies that make medications convenient, provide education and reminders as needed, offer feedback on progress, and provide a therapeutic relationship. ${ }^{1,12,13}$

Appointment-based medication synchronization (ABMS) has been promoted as an effective strategy to improve patient adherence and persistence with medication regimens. ${ }^{14,15}$ ABMS was first implemented in 1995 by a California pharmacist, John Sykora, to standardize medication dispensing schedules to improve medication management and adherence. ABMS is increasingly being offered by pharmacies as part of the patient-centric and medication synchronization models and as specific programs such as Sync Your Meds and Simplify My Meds. Specific details often vary between programs but the basic ABMS process consists of 3 key features: ${ }^{14}$

1. Pharmacists working with patients to synchronize medication refills to come due on a single day of the month.

2. A regular call (e.g., monthly) from the pharmacy to the patient or designated care provider to identify which medications to fill. This call typically occurs 5 to 7 days prior to the scheduled pharmacy visit and consists of an opportunity to clarify changes in therapy, address managed care issues such as prior authorizations, and engage in mutual problem solving regarding nonadherence issues. This call differs from automatic refill programs, which refill prescriptions on a scheduled basis and notify patients when the prescriptions are ready. With automatic refills, contact with the pharmacy is minimized, leaving little opportunity to proactively address adherence issues.

3. An appointment date where patients are scheduled to pick up medications or have them delivered. On this date, the medications can simply be dispensed or they can be accompanied by medication therapy management, disease management, or other more intensive pharmacist-delivered services.

A study of an ABMS program in rural pharmacies in the midwestern United States indicated that the program was associated with greater patient adherence and persistence when patients first start their chronic medications..$^{15}$ Patients enrolled in ABMS who received medications in 6 different chronic disease categories had 3.4 to 6.1 times greater odds of adherence over a 1-year period than matched comparisons. Individuals who were not enrolled were at least 50\% more likely to stop taking their chronic medications over 1 year. To date, it is not known whether similar results would be seen for patients who have been taking chronic medications over time.
The objective of this analysis was to compare the effect on medication adherence and persistence of an ABMS program in community pharmacies with standard of care. Dispensing records were obtained for patients receiving medications from at least 1 of 6 medication categories: angiotensin-converting enzyme inhibitors or angiotensin receptor blockers, beta blockers, dihydropyridine calcium channel blockers, thiazide diuretics, metformin, and statins. These records were compared between ABMS and matched comparisons. The medication categories in this study were chosen because of their widespread use, history of nonadherence and persistence in patients, and importance in achieving therapeutic outcomes when taken as directed. ${ }^{2,3,9}$ It was hypothesized that existing medication users who enrolled in the ABMS program would have higher adherence and persistence than patients who were not in the ABMS (matched comparison) group.

\section{Methods}

\section{Study Design}

This retrospective cohort study was conducted on patients served by Discount Drug Mart, Inc., an Ohio-based drugstore chain of 71 pharmacies in 20 Ohio counties. Data for this study came from prescription claims records of commercially insured individuals collected from December 1, 2011, to February 28, 2014. All ABMS patients received 2 or more consecutive fills for 1 of 6 chronic medication classes after enrollment and were followed for 1 year. ABMS patients were matched with comparison patients receiving usual care according to their histories of prior adherence behavior (PAB), medication class, age, gender, and geographic region. Individuals in the matched comparison group included individuals who opted out of the program or were never asked to enroll. The study was approved by the Institutional Review Board of Virginia Commonwealth University.

\section{Enrollment}

Individuals "opted in" to ABMS after being informed about the availability and details of the program by a clerk, technician, or pharmacist. Although any patient could enroll, patients with multiple, ongoing monthly prescriptions for chronic disease conditions were targeted, especially if gaps in refill patterns were noticed. All enrolled individuals were required to read, understand, and sign an ABMS Patient Agreement Form that reviewed the features of the program and patient requirements to (a) accept a monthly phone call from the pharmacy to discuss prescription refills, (b) have ABMS prescriptions filled with a 1-month supply, (c) pick up medications on a mutually agreed upon assigned appointment date, (d) pay any additional copays needed to synchronize all refills to be due on the same day, (e) have an open dialogue with the pharmacist about physician appointments, hospital/urgent care visits, and changes in health status, and ( $\mathrm{f}$ ) give permission for the pharmacist 
to discuss details of the program with the patient's caregiver. Patients who did not consent to the ABMS agreement were excluded from the program.

\section{Intervention}

After patient consent and enrollment, 3 actions occurred in ABMS: synchronization of medications, a monthly call to the patient or family caregiver, and a scheduled appointment to pick up medication at the pharmacy and speak with the pharmacist as needed. In the synchronization phase, the patient and pharmacist chose a monthly appointment day to pick up prescriptions. This one-time "synchronization" typically consisted of the pharmacist reviewing the patient's medication profile, identifying an "anchor" prescription to which all other prescriptions would be synchronized, and determining the quantity needed for all other prescriptions to be refilled with the anchor prescription. Another part of synchronization was a contact to the patient's physician to explain ABMS and ask for a one-time prescription for the quantity required for each medication to be synchronized to the anchor.

After synchronization, a call was made approximately 7 days prior to the appointment day. During this call, the pharmacist reviewed the patient's medication list, asked about any new or changed prescriptions, and confirmed which prescriptions needed to be filled. Discussions also occurred about physician and hospital visits and any issues with the patient's medications. If necessary, the physician was contacted to resolve any discrepancies.

On the date of the appointment visit to the pharmacy, an automated outbound phone call was made to inform the patient that the medications were ready to be picked up. The medications were typically filled 3 days prior to the appointment to give time for the pharmacist to troubleshoot any problems with insurance, inventory shortages, or other issues. When the patient arrived to pick up the medications, the medications were dispensed like any other prescription, or the appointment might be combined with some other medication therapy management service, such as a comprehensive medication review.

\section{Measures}

The outcomes used to assess ABMS were adherence and nonpersistence over a l-year period. Adherence was measured using proportion of days covered (PDC), and patients were considered adherent with a PDC of at least $0.80 .{ }^{16}$ Nonpersistence was calculated by identifying the date at which a patient stopped taking a medication within the chronic medication category for 30 days or more. At that point, they were labeled nonpersistent.

\section{Data Analysis}

The analysis period for this study was from March 1, 2013, to February 28, 2014 (Figure 1). All prescription claims were lim- ited to a 30-day supply. Since all ABMS synchronized patients were already using the medications at the time of enrollment (i.e., no new users), a 6-month pre-enrollment period for each study patient was used to assess PAB. This 6-month period varied for each patient, depending on when the patient enrolled in the program. Only patients who started the program sometime between December 1, 2011, and the start of the 1-year analysis period, March 1, 2013, were analyzed because 6 months of data prior to that period were not available. Data availability also prevented analysis of any differences in how long patients had been taking their medications. PAB in comparison patients was calculated from data collected in the same time periods.

Measures for the ABMS program were compared with usual care. Up to 3 comparison patients per study patient were used to match on PAB, medication class, age, gender, and geographic region using a 2-stage process. Patients were first categorized according to medication class, so patients taking multiple medications could fall into more than 1 class. Patients were then matched within each medication class on age, gender, geographical region, and PAB score. This allowed comparisons of adherence and persistence according to medication use classes. Neither pill burden nor medication use outside of the 6 medication classes were examined in the study. The number of patients in the comparison group who were offered but refused enrollment into the program was not measured. In the original design, sulfonylureas were included in the data analysis, but because of low sample sizes after matching ( $\mathrm{n}=31$ in usual care; $\mathrm{n}=35$ among ABMS patients), this medication class was excluded from the final analysis.

PAB scores were calculated to compare differences in history of medication use between ABMS patients and usual care. ${ }^{17,18}$ These scores were constructed using 10 levels based upon patients' PDC scores for the 6-month pre-enrollment period $(<0.10,0.10-0.19$, $0.20-0.29,0.30-0.39,0.40-0.49,0.50-0.59,0.60-0.69,0.70-0.79$, 0.80-0.89, and >0.90). ABMS and comparison patients were assigned PAB scores, which were used in matching.

Descriptive statistics and statistical comparisons of adherence and nonpersistence were conducted. Statistical comparisons of PDC between groups used the Friedman test to account for the paired nature of the matched comparisons and the non-normality of the PDC. Comparisons of the proportion of adherent patients in groups were assessed with a univariate conditional logistic regression, which provided the odds of adherence by study and comparison group. Because no dropout date was provided, patients who dropped out could not be censored. Hence, using the intention-to-treat principle, patients who dropped out were assumed to continue with the program as a conservative measure, and time of medication discontinuation was analyzed with a univariate conditional Cox proportional hazards regression. A $P$ value of $<0.05$ was considered statistically significant. The data analysis was conducted using SAS software, version 9.4 (SAS Institute Inc., Cary, NC). 


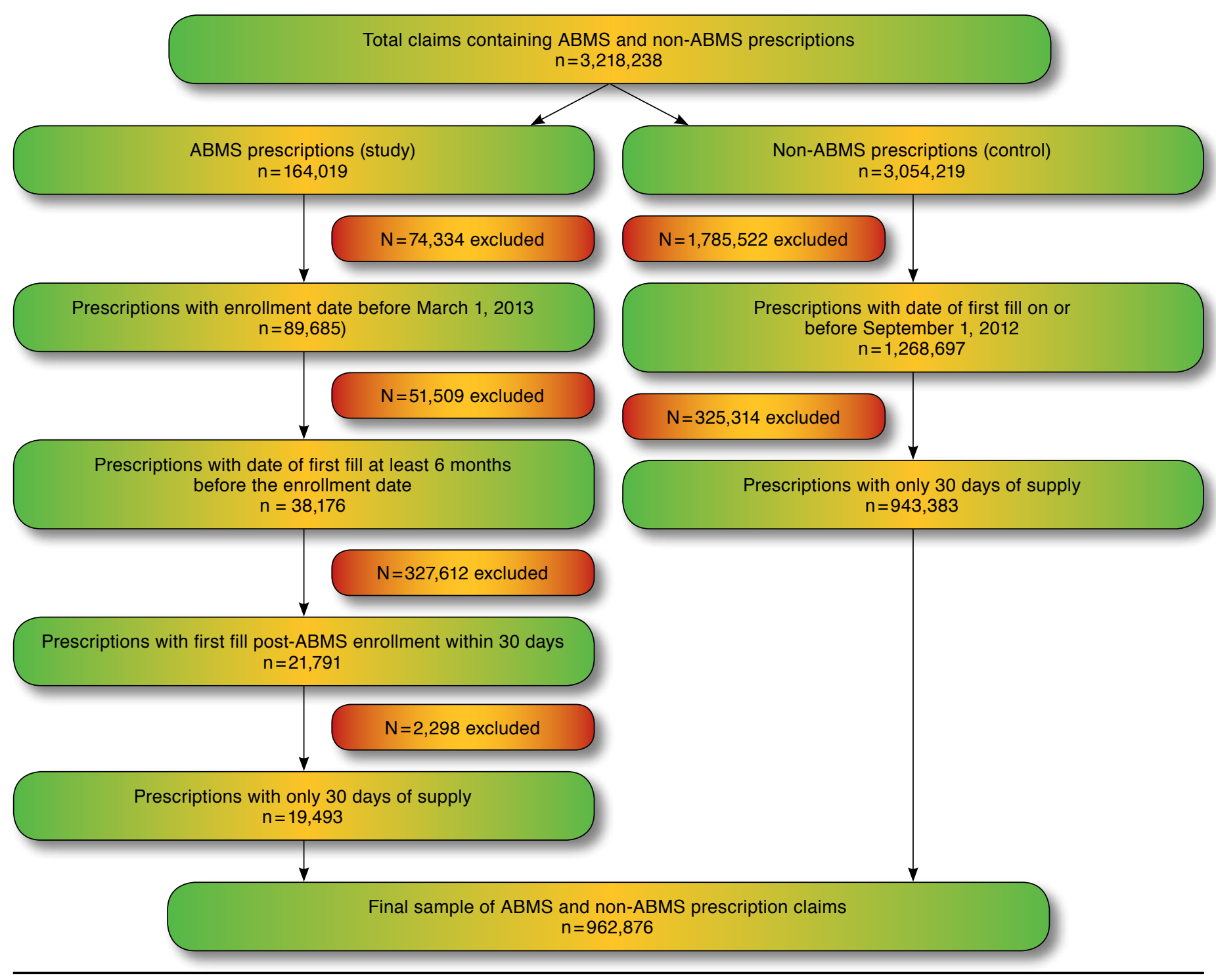

ABMS = appointment-based medication synchronization.

\section{Results}

Descriptive information about patients and their PAB is presented in Table 1 and Figure 2, respectively. The average patient was female, between aged 59 and 66 years, and had some form of prescription insurance coverage. Because of matching, ABMS and usual care were similar in PAB, medication class, age, gender, and geographic region. There was little difference between the groups in plan type. Final sample sizes for ABMS and comparison patients ranged from 140 to 1,133 (Table 2).

Medication adherence differed between ABMS and usual care prior to the start of the study (Figure 2). This finding was anticipated based upon an expectation that patients who were predisposed to be adherent would be more likely to enroll in adherence programs. This appeared to be the case because the percentage of adherent patients as measured by PAB scores was more than 17 points higher for ABMS patients for all medication categories before enrolling in the program.

Nevertheless, mean PDC scores for ABMS patients in all drug classes were significantly greater than those for usual care after 1 year even after matching for PAB. Mean PDCs for the comparison group ranged from 0.57 to 0.71 , while those for patients in the ABMS program ranged from 0.73 to 0.91 . 


\begin{tabular}{|c|c|c|c|c|c|c|}
\hline Variable & ACEIs/ARBs & Beta Blockers & DCCBs & Thiazide Diuretics & Metformin & Statins \\
\hline Total & 37,189 & 27,251 & 15,427 & 23,801 & 7,373 & 33,754 \\
\hline Age (years), mean (SD) & $62.6(14.8)$ & $64.2(16.2)$ & $66.1(15.7)$ & $64.6(16.4)$ & $59.2(14.7)$ & $63.7(13.2)$ \\
\hline Female (\%) & 51.5 & 54.6 & 57.69 & 63.3 & 55.5 & 51.6 \\
\hline Number of ABMS patients & 703 & 544 & 326 & 499 & 205 & 716 \\
\hline Number of controls & 36,486 & 26,707 & 15,101 & 23,302 & 7,168 & 33,038 \\
\hline \multicolumn{7}{|l|}{ Plan type (\%) } \\
\hline Cash & 19.2 & 17.6 & 17.0 & 18.5 & 19.3 & 15.9 \\
\hline Third party & 80.8 & 82.4 & 83.0 & 81.5 & 80.7 & 84.1 \\
\hline
\end{tabular}

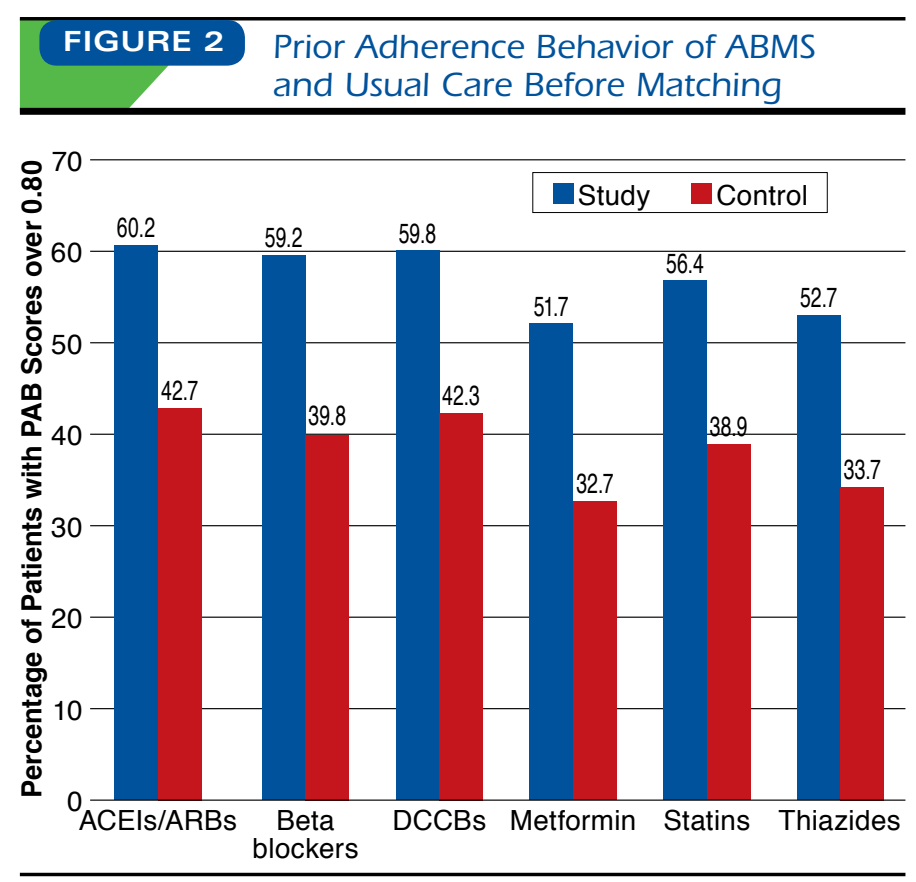

ABMS = appointment-based medication synchronization; $A C E I=$ angiotensin-converting enzyme inhibitor; $A R B=$ angiotensin receptor blocker; $D C C B=$ dihydropyridine calcium channel blocker; $P A B=$ prior adherence behavior.

The percentage of adherent patients (i.e., $\mathrm{PDC} \geq 0.80$ ) was significantly greater for ABMS patients compared with usual care (Table 3). In the comparison group, approximately $37 \%$ to $62 \%$ were found to be adherent depending on the drug class. In contrast, the percentage of adherent patients in the ABMS program ranged from 55\% to $84 \%$. When comparing differences between groups, approximately 18 to 35 additional individuals were adherent in the ABMS group for every 100 patients depending on drug class. When evaluating the odds of adherence, patients enrolled in the program had 2.3 to 3.6 times greater odds of adherence (depending on drug class) compared with usual care.

\section{TABLE 2 Proportion of Days Covered by Drug Class}

\begin{tabular}{|c|c|c|c|c|c|c|c|}
\hline \multirow[b]{2}{*}{ Drug Class } & \multicolumn{3}{|c|}{ Usual Care } & \multicolumn{3}{|c|}{ ABMS Patients } & \multirow[b]{2}{*}{$\begin{array}{c}P \\
\text { Value }\end{array}$} \\
\hline & $\mathrm{n}$ & $\begin{array}{c}\text { Mean } \\
\text { PDC }\end{array}$ & SD & $\mathrm{n}$ & $\begin{array}{c}\text { Mean } \\
\text { PDC }\end{array}$ & SD & \\
\hline ACEIs/ARBs & 1,133 & 0.71 & 0.301 & 584 & 0.91 & 0.225 & $<0.001$ \\
\hline Beta blockers & 856 & 0.64 & 0.324 & 452 & 0.78 & 0.277 & $<0.001$ \\
\hline DCCBs & 418 & 0.62 & 0.329 & 246 & 0.81 & 0.288 & $<0.001$ \\
\hline Thiazide diuretics & 687 & 0.58 & 0.341 & 385 & 0.77 & 0.284 & 0.001 \\
\hline Metformin & 232 & 0.57 & 0.338 & 140 & 0.73 & 0.280 & 0.006 \\
\hline Statins & 1,197 & 0.63 & 0.313 & 600 & 0.80 & 0.259 & $<0.001$ \\
\hline \multicolumn{8}{|c|}{$\begin{array}{l}\text { ABMS = appointment-based medication synchronization; } A C E I=\text { angiotensin- } \\
\text { converting enzyme inhibitor; } A R B=\text { angiotensin receptor blocker; } D C C B=\text { dihydro- } \\
\text { pyridine calcium channel blocker; } P D C=\text { proportion of days covered; } S D=\text { standard } \\
\text { deviation. }\end{array}$} \\
\hline
\end{tabular}

The percentage considered nonpersistent according to group and hazard ratios are displayed in Table 4. In the comparison group, approximately $61 \%$ to $74 \%$ became nonpersistent within 1 year after starting, while 33\% to $44 \%$ became nonpersistent in the ABMS group. When comparing persistence between groups for every 100 individuals, approximately 17 to 40 additional individuals remained persistent for 1 year in the ABMS group. Compared with usual care, ABMS patients had a $33 \%$ to $61 \%$ lower likelihood of nonpersistence, depending on the drug class.

\section{Discussion}

This study provides evidence for the effectiveness of ABMS programs in community pharmacy settings for improving adherence and persistence with chronic medications. Patients enrolled in ABMS programs were significantly more adherent and persistent with medicines compared with the standard of care when controlling for PAB, medication class, age, gender, and geographic region. The findings were consistent across all 6 drug classes studied. 


\begin{tabular}{|c|c|c|c|c|c|c|}
\hline \multirow[b]{3}{*}{ Drug Class } & \multicolumn{6}{|c|}{$\begin{array}{l}\text { Percentage of Patients Adherent } \\
\text { and Odds Ratios from Univariate } \\
\text { Logistic Regression }\end{array}$} \\
\hline & \multicolumn{2}{|c|}{ Adherent (\%) } & \multirow{2}{*}{$\begin{array}{l}\text { Increase in } \\
\% \text { Adherent } \\
\text { with ABMS }\end{array}$} & \multirow[b]{2}{*}{ OR } & \multirow[b]{2}{*}{$(95 \%$ CI $)$} & \multirow[b]{2}{*}{$\begin{array}{c}P \\
\text { Value }\end{array}$} \\
\hline & $\begin{array}{l}\text { Usual } \\
\text { Care }\end{array}$ & ABMS & & & & \\
\hline ACEIs/ARBs & 62.0 & 84.3 & 22.3 & 3.3 & \begin{tabular}{|l|}
$1.7-4.1$ \\
\end{tabular} & $<0.001$ \\
\hline Beta blockers & 44.5 & 66.4 & 21.9 & 3.1 & $2.4-4.1$ & $<0.001$ \\
\hline DCCBs & 43.3 & 70.3 & 27.0 & 3.4 & $2.3-5.0$ & $<0.001$ \\
\hline Thiazide diuretics & 39.5 & 74.2 & 34.7 & 3.3 & $2.4-4.5$ & $<0.001$ \\
\hline Metformin & 36.6 & 55.0 & 18.4 & 2.3 & $1.3-3.9$ & 0.003 \\
\hline Statins & 41.8 & 66.7 & 24.9 & 3.6 & $2.8-4.6$ & $<0.001$ \\
\hline \multicolumn{7}{|c|}{$\begin{array}{l}\text { aPercentage of individuals with } P D C>80 \% . \\
\text { ABMS =appointment-based medication synchronization; } A C E I=\text { angiotensin- } \\
\text { converting enzyme inhibitor; } A R B=\text { angiotensin receptor blocker; } C I=\text { confidence } \\
\text { interval; } D C C B=\text { dihydropyridine calcium channel blocker; } O R=\text { odds ratio; } \\
P D C=\text { proportion of days covered. }\end{array}$} \\
\hline
\end{tabular}

This study makes an important contribution in the area of medication adherence and persistence by examining the impact of medication synchronization on a different population than past research. In contrast to other research, which looked at the effect of ABMS on patients who were first starting chronic medications, this study examined patients who had been taking chronic medications for at least 6 months. ${ }^{14,15}$

This patient population is significant because nonadherence is more likely to occur in patients who fill new prescriptions, with approximately 50\% discontinuing therapy in the first 6 months., ${ }^{5,912}$ Excluding these relatively nonadherent patients from the analysis makes the overall study population more adherent and thereby more difficult to improve upon. In other words, marginal improvements in adherence are harder with adherent populations. This study showed that ABMS works on these patients, too.

Another contribution of this study was controlling for previous adherence behavior between ABMS patients and usual care. Only a few previous studies have controlled for the "healthy adherer" effect, a situation in which patients who are predisposed to take medicines more consistently will be more likely to enroll in adherence programs. ${ }^{17-19}$ This study matched groups using $\mathrm{PAB}$ scores to control for the healthy adherer effect.

Figure 2 demonstrates potential evidence for the healthy adherer effect in the studied population. Patients who enrolled in the ABMS program were much more adherent at the beginning of the study than comparison groups-more than 17 percentage points in all 6 groups. When this effect was reduced by matching according to PAB scores, patients in the ABMS program still showed significantly higher adherence and persistence than the comparison groups.

The ABMS program may be effective because it is a complex intervention consisting of a variety of opportunities to

\begin{tabular}{|c|c|c|c|c|c|c|}
\hline \multirow[b]{2}{*}{ Drug Class } & \multicolumn{2}{|c|}{\begin{tabular}{|c|} 
Nonpersistence \\
$(\%)$
\end{tabular}} & \multirow{2}{*}{$\begin{array}{l}\text { Difference } \\
\text { in Non- } \\
\text { persistence } \\
\text { with ABMS }\end{array}$} & \multirow[b]{2}{*}{ HR } & \multirow[b]{2}{*}{$(95 \% \mathrm{CI})$} & \multirow[b]{2}{*}{$\begin{array}{c}P \\
\text { Value }\end{array}$} \\
\hline & $\begin{array}{l}\text { Usual } \\
\text { Care }\end{array}$ & ABMS & & & & \\
\hline ACEIs/ARBs & 65.8 & 32.7 & -33.1 & 0.46 & $0.35-0.56$ & $<0.001$ \\
\hline Beta blockers & 68.2 & 40.3 & -27.9 & 0.59 & $0.46-0.67$ & $<0.001$ \\
\hline DCCBs & 72.4 & 39.1 & -33.3 & 0.42 & $0.37-0.55$ & $<0.001$ \\
\hline Thiazide diuretics & 69.6 & 42.5 & -27.1 & 0.45 & $0.36-0.53$ & $<0.001$ \\
\hline Metformin & 60.8 & 43.7 & -17.1 & 0.67 & $0.51-0.75$ & 0.009 \\
\hline Statins & 74.2 & 33.8 & -40.4 & 0.39 & $0.29-0.46$ & $<0.001$ \\
\hline
\end{tabular}

influence how patients take their medications. The first is the enrollment process, which requires each patient to commit to actively participating in medication therapy. The commitment to enroll can increase the likelihood that patients will take their medications. ${ }^{20}$ The ABMS program also provides regular opportunities for the pharmacist, patient, and physician to communicate and solve problems, which are key activities for improving medication adherence and persistence. ${ }^{21,22}$ Finally, ABMS reminds patients to pick up their prescriptions and simplifies the physical acquisition of medications. It is not clear if the findings of this study would be duplicated for programs that do not have all of the elements of ABMS. Similar to a medication, a minor change in the content of the ABMS program may alter its overall effectiveness in patients.

In addition to the clinical benefits of ABMS, the findings of this research have important financial implications for health plans and pharmacies. Significant improvements in adherence can help Medicare Advantage plans improve their Medicare star ratings, which may make them eligible for performance bonuses and rebates. Medication adherence of plan members is an important factor in star ratings, and improving adherence for the medications in this research-diabetic medications, statin therapies, and hypertension drugs-can increase compensation.

Pharmacies can benefit from ABMS in a variety of ways. On the revenue side, ABMS can reduce lost sales due to nonadherence and help pharmacies make a strong case for having access to patients in preferred pharmacy networks. Pharmacies that show the ability to drive patient medication adherence and persistence will be able to demonstrate their value to health plan administrators. On the cost side, ABMS can help pharmacists manage inventory, improve workflow, and manage personnel costs better. No evidence is currently available about the real financial benefits of ABMS, but the economic logic is persuasive. 


\section{Limitations}

There are some limitations associated with the findings of this study. The findings of the current work may not be duplicated in other settings, although research indicates external validity for the results. A study examining the ABMS program on a different patient population located in another geographic setting showed consistent results with our study. ${ }^{3}$ In addition, a white paper summarizing other medication synchronization programs also indicated support for the effectiveness of ABMS in a broad range of geographic and community settings. ${ }^{4}$

This analysis did not control for all factors affecting adherence to medications. Factors not addressed in the study design included patient insurance status, complexity of medication regimens, severity of conditions, and level of patient motivation and engagement in health care. It also used pharmacy prescription fill data, which may not reflect how patients actually take their medications. In addition, the adherence and persistence measures in this study do not indicate whether the patients actually take the medications they pick up or take them as directed. It is possible that some patients simply stock pile their medications or take them inappropriately.

The possibility of self-selection bias is another limitation. The people who sign up for an ABMS program might be inherently different from those who do not (e.g., level of engagement or interest in health care). Also, some patients labeled as nonpersistent may have simply switched pharmacies. These limitations were not addressed in the design of this study.

\section{Conclusions}

This is the first study to assess the impact of ABMS on community pharmacy patients who have been taking their chronic medications for at least 6 months. The ABMS program is associated with improved patient adherence and reduced likelihood of nonpersistence.

One might expect further improvements in results over time, since this is a study of a new program in which pharmacists are just learning how to integrate it into their systems. As methods become more standardized and best practices are learned and implemented through continuous quality improvement, the impact of ABMS may improve as experience is gained.

\section{Authors}

DAVID HOLDFORD, RPh, MS, PhD, FAPhA, is Professor of Pharmacoeconomics and Health Outcomes, and KUNAL SAXENA, BS, MS, is a PhD Candidate, Department of Pharmacotherapy $E$ Outcomes Science, Virginia Commonwealth University School of Pharmacy, Richmond, Virginia.

AUTHOR CORRESPONDENCE: David Holdford, RPh, MS, PhD, FAPhA, McGuire Hall, Rm. 213, P.O. Box 980533, 1112 E. Clay St., Richmond, VA 23298-0533. Tel.: 804.828.6103;

Fax: 804.628.3991; E-mail: daholdfo@vcu.edu.

\section{DISCLOSURES}

Funding for this study was contributed by Pfizer. The authors had complete control over preparation, writing, revision, and approval of this manuscript. The authors declare no conflict of interest.

Study concept and design were primarily contributed by Holdford along with Saxena. Saxena was primarily responsible for data collection and interpretation, along with Holdford. Manuscript writing and revision was performed primarily by Holdford, along with Saxena.

\section{REFERENCES}

1. Sabate E, ed. Adherence to long-term therapy: evidence for action. World Health Organization. 2003. Available at: http://whqlibdoc.who.int/publications/2003/9241545992.pdf. Accessed June 24, 2015.

2. Wong MC, Tam WW, Cheung CS, et al. Drug adherence and the incidence of coronary heart disease- and stroke-specific mortality among 218,047 patients newly prescribed an antihypertensive medication: a fiveyear cohort study. Int J Cardiol. 2013;168(2):928-33.

3. Rasmussen JN, Chong A, Alter DA. Relationship between adherence to evidence-based pharmacotherapy and long-term mortality after acute myocardial infarction. JAMA. 2007;297(2):177-86.

4. Sokol MC, McGuigan KA, Verbrugge RR, Epstein RS. Impact of medication adherence on hospitalization risk and healthcare cost. Med Care. 2005;43(6):521-30.

5. Osterberg L, Blaschke T. Adherence to medication. N Engl J Med. 2005;353(5):487-97.

6. Cutler DM, Everett W. Thinking outside the pillbox-medication adherence as a priority for health care reform. N Engl J Med. 2010;362(17):1553-55.

7. Dimatteo MR. Variations in patients' adherence to medical recommendations: a quantitative review of 50 years of research. Med Care. 2004;42(3):200-09.

8. Benner JS, Glynn RJ, Mogun H, Neumann PJ, Weinstein MC, Avorn J. Long-term persistence in use of statin therapy in elderly patients. JAMA. 2002;288(4):455-61.

9. Cramer JA, Benedict A, Muszbek N, Keskinaslan A, Khan ZM. The significance of compliance and persistence in the treatment of diabetes, hypertension and dyslipidaemia: a review. Int J Clin Pract. 2008;62(1):76-87.

10. Avorn J, Monette J, Lacour A, et al. Persistence of use of lipid-lowering medications: a cross-national study. JAMA. 1998;279(18):1458-62.

11. Briesacher BA, Andrade SE, Fouayzi H, Chan KA. Comparison of drug adherence rates among patients with seven different medical conditions. Pharmacotherapy. 2008;28(4):437-43.

12. Haynes RB, Ackloo E, Sahota N, McDonald HP, Yao X. Interventions for enhancing medication adherence. Cochrane Database Syst Rev. 2008;(2):CD000011.

13. Viswanathan $\mathrm{M}$, Golin $\mathrm{CE}$, Jones $\mathrm{CD}$, et al. Interventions to improve adherence to self-administered medications for chronic diseases in the United States: a systematic review. Ann Intern Med. 2012;157(11):785-95.

14. American Pharmacists Association Foundation. Pharmacy's appointment based model: a prescription synchronization program that improves adherence. APhA Foundation White Paper. August 30, 2013. Available at: http:// www.aphafoundation.org/sites/default/files/ckeditor/files/ABMWhitePaperFINAL-20130923(3).pdf. Accessed June 24, 2015.

15. Holdford DA, Inocencio TJ. Adherence and persistence associated with an appointment-based medication synchronization program. J Am Pharm Assoc (2003). 2013;53(6):576-83.

16. Pharmacy Quality Alliance. PQA performance measures. Updated May 2015. Available at: http://pqaalliance.org/measures/default.asp. Accessed June 24, 2015 
17. Iyengar R, Henderson R, Visaria J, Glave FS. Dispensing channel and medication adherence: evidence across 3 therapy classes. Am J Manag Care. 2013;19(10):798-804.

18. Iyengar RN, Balagere DS, Henderson RR, LeFrancois AL, Rabbitt RM, Frazee SG. Association between dispensing channel and medication adherence among medicare beneficiaries taking medications to treat diabetes, high blood pressure, or high blood cholesterol. J Manag Care Spec Pharm. 2014;20(8):851-61. Available at: http://www.amcp.org/JMCP/2014/ August/18390/1033.html.
19. Patrick AR, Shrank WH, Glynn RJ, et al. The association between statin use and outcomes potentially attributable to an unhealthy lifestyle in older adults. Value Health. 2011;14(4):513-20.

20. Putnam DE, Finney JW, Barkley PL, Bonner MJ. Enhancing commitment improves adherence to a medical regimen. J Consult Clin Psychol. 1994;62(1):191-94.

21. Haskard Zolnierek KB, DiMatteo MR. Physician communication and patient adherence to treatment: a meta-analysis. Med Care. 2009;47(8):826-34.

22. Brunton SA. Improving medication adherence in chronic disease management. J Fam Pract. 2011;60(4 Suppl Improving):S1-S8. 